\title{
Surface Identification Using Satellite Microwave Radiometers
}

\author{
NORMAN C. GRODY
}

\begin{abstract}
The use of satellite microwave radiometers for identifying natural surfaces is analyzed. A retrieval technique is developed by considering the related "mixed pixel" problem where two or more surfaces are contained within the viewing area. At frequency $w$ the emissivity measurement $\epsilon(w)$ depends on the fractional amounts $f_{n}$ and $a$ priori emissivities $\epsilon_{n}(w)$ where $\epsilon(w)=\Sigma \epsilon_{n}(w) f_{n}$. In applications involving surface identification the fractional amounts act as discriminants to identify the most likely surface among the $a$ priori candidates. In principle the fractional amounts can be obtained using multispectral measurements of emissivity. However, due to the limited spectral characteristics of emissivity the maximum number of distinguishable surfaces is reduced to three. The fractional amounts are derived using dual-frequency emissivity measurements and the effects of errors in measurement and a priori values are analyzed.
\end{abstract}

\section{INTRODUCTION}

M ICROWAVE radiometers have been flown aboard satellites for the past 20 years. A historical summary is presented in the introductory volume by Ulaby $e t$ al. [1] (see pp. 14-15 for summary table) and a comprehensive review of microwave applications is given in vol. 3 of the series [2] (see ch. 17 for atmospheric applications and chs. 18 and 19 for surface measurements). Depending on the application, the spatial resolutions have ranged between 20 and $200 \mathrm{~km}$ for the different instruments. These radiometers contain channels within the $50-60 \mathrm{GHz}$ region of the oxygen band, around the $22-\mathrm{GHz}$ water vapor line, as well as within the more transparent spectral regions of the atmosphere. The oxygen and water vapor channels are used to derive temperature profiles and water vapor content [2]-[4] while the "window" channels provide surface and precipitation information [2], [5], [6]. Of all the parameters, precipitation and surface measurements require the highest resolution. Precipitation also demands the most frequent observations. The cloud penetrating property of microwaves enables these atmospheric and surface parameters to be obtained globally independent of cloud cover using measurements from the different spectral regions.

One of the most challenging problems concerns the application of microwave radiometers to surface measurements. The major differences between surfaces is obtained from their emissivity spectra. In some cases the polarization measurements of emissivity can provide ad-

Manuscript received May 21, 1987; revised June 7, 1988

The author is with the National Oceanic and Atmospheric Administration, Washington, DC 20233.

IEEE Log Number 8823382 ditional information on surface conditions [2]. Also useful is the spatial and temporal variation of emissivity [7]. Further improvements are sometimes possible using $a$ priori climatological data, although the variability can be quite large for surfaces. This paper only considers the use of multispectral measurements to identify and retrieve surface parameters. Analyses of the accuracy and limiting factors are presented.

\section{Surface Identification as a Retrieval Problem}

Microwave radiometers aboard satellites measure the thermal emission from the Earth's surface and intervening atmosphere. For channels that respond to the surface (window channels) the brightness temperature $T_{b}$ can be approximated by considering an isothermal atmosphere [4] so that

$$
T_{b}(w)=T_{s}\left[1-\tau_{w}^{2}(1-\epsilon(w))\right]
$$

where $\tau$ is the atmospheric transmittance, $T_{s}$ is the surface temperature, and $\epsilon$ is the surface emissivity. The transmittance and emissivity generally depend on the observation frequency and viewing angle. Only the frequency dependence $w$ is indicated in (1). Emissivity also depends on the polarization of emitted radiation.

To estimate the error in the emissivity measurement $\delta \epsilon$, consider a single frequency measurement having errors in transmittance $\delta \tau$, surface temperature $\delta T_{s}$, and brightness temperature noise $\delta T_{b}$. By differentiating (1) the emissivity error ( $\mathrm{rms}$ ) is given by

$$
\begin{aligned}
\sqrt{\left\langle\delta \epsilon^{2}\right\rangle}= & {\left[\left\langle\delta T_{b}^{2}\right\rangle+\left(T_{b} / T_{s}\right)^{2}\left\langle\delta T_{s}^{2}\right\rangle\right.} \\
& \left.+4(1-\epsilon) T_{s}\left(T_{s}-T_{b}\right)\left\langle\delta \tau^{2}\right\rangle\right]^{1 / 2} / \tau^{2} T_{s}
\end{aligned}
$$

where the errors in transmittance, brightness temperature, and surface temperature are assumed independent random variables. The component due to transmittance error is minimized for high emissivity land surfaces. This term is also negligible at low frequencies $(<10 \mathrm{GHz})$ and at higher frequencies in the polar regions where the absorption due to clouds and water vapor is very small. For these conditions the emissivity error depends on surface temperature, where an uncertainty of ten degrees can result in a three-percent error in emissivity. Errors in the emissivity measurement due to atmospheric effects or surface temperature variations are generally highly correlated for 
adjacent channels. As discussed later, the emissivity error has a direct influence on the problem of surface identification, which is the main subject of this paper.

The interpretation of surface measurements is complicated by the resolution of microwave radiometers, which generally exceeds $20 \mathrm{~km}$ from a space platform. Even smaller fields of view can average many surface features within a single observation (pixel). This is referred to as the "mixed pixel" problem and was evident in the measurements obtained by the Scanning Multichannel Microwave Radiometer (SMMR) on the Nimbus 7 satellite [5]. Unlike oceans, over land the largest variations in brightness temperature (1) are generally associated with the emissivity, which is a spatially averaged quantity. For the purpose of analysis the field of view is subdivided into a finite number of surfaces and the emissivity is given by

$$
\epsilon(w)=\sum_{n=0}^{N} \epsilon_{n}(w) f_{n}
$$

where

$$
\sum_{n=0}^{N} f_{n}=1 .
$$

The functions $\epsilon_{n}(w)$ represent the emissivities for the different surfaces (water, snow, ice, etc.,) within the viewing area, where each surface has a fractional amount $f_{n}$ whose sum is unity. Note that the mixed pixel problem has been reduced to that of determining the percent concentrations $f_{n}$, using multifrequency measurements $\epsilon(w)$. Furthermore, in the case of a single unknown surface, (3a) can be viewed as an expression of its emissivity in terms of known functions having undetermined coefficients. In applications involving surface identification the fractional amounts serve as a discriminant to identify the most likely surface among the a priori candidates. In this case the constraint on the coefficients given by ( $3 b)$ serves to normalize the emissivity to values between say open water, which usually has the lowest emissivity, and dry land, which has the largest emissivity. As with any inverse problem, the accuracy of the retrieved coefficients depends on the measurement error as well as the uncertainty and uniqueness of the a priori elements $\epsilon_{n}(w)$.

In setting up the retrieval problem the number of terms in ( $3 a$ ) is reduced by one using (3b), viz.

$$
E(w)=\sum_{n=1}^{N} W_{n}(w) f_{n}
$$

where

$$
E(w)=\epsilon(w)-\epsilon_{0}(w)
$$

and

$$
W_{n}(w)=\epsilon_{n}(w)-\epsilon_{0}(w) .
$$

The determination of $f_{n}$ formally reduces to the inversion of the matrix $W_{n}(w)$. However, before proceeding it is necessary to examine the characteristics of the matrix elements, or more explicitly, the frequency dependence of emissivity for different surfaces. It should be noted that the formulation can be expanded to also include the use of polarization measurements; however, this will be the subject of future papers.

\section{Emissivity Characteristics}

During the past decade there have been many theoretical and experimental investigations of the emissivity properties at microwave frequencies [2], [8]. The following is a brief description of the emissivity characteristics for different surfaces.

Fig. 1 displays the emissivity at nadir as a function of frequency for various surfaces. The emissivity of wet soil is obtained from model calculations using a soil moisture of $0.4 \mathrm{~cm}^{3} / \mathrm{cm}^{3}$ with an average wilting point of 0.2 $\mathrm{cm}^{3} / \mathrm{cm}^{3}$ [9]. Calculations are also used to obtain the sea surface emissivity [10]. In the case of sea ice and snow the models become most uncertain so that aircraft and ground-based measurements are used [11], [12]. The overall frequency response is obtained by fitting the data (at widely separated frequencies) to the empirical function

$$
\epsilon_{n}(w)=\frac{\epsilon_{0}+\epsilon_{\infty}(w / w 0)^{k}}{1+(w / w 0)^{k}}
$$

where the parameters $\epsilon_{0}, \epsilon_{\infty}, w 0$, and $k$ are listed in Table I. Plots of the function and the corresponding measurements are shown in Fig. 1. The frequency characteristics of this empirical model are that of a low or high pass filter depending on whether the material acts more as an absorber or a scatterer of radiation, respectively. For a scattering material $\epsilon_{0}$ is greater than $\epsilon_{\infty}$, and the parameter $w 0$ denotes the lowest frequency at which scattering becomes important. Also, the parameter $k$ is proportional to the slope of emissivity with frequency near $w 0$. It should be mentioned that the smaller scale variations due to stratification in snow and ice are not included in this four parameter model. More detailed frequency measurements of emissivity are required to identify and model these effects.

As shown in Fig. 1, for homogeneous materials such as water, soil, and newly formed ice, the emissivity varies little or increases with frequency. Also, because water has the highest dielectric constant, its emissivity is the lowest value. Consequently, dry soil, which contains little water, has the largest emissivity. Depending on the soil moisture, the emissivity can decrease to that of a free water surface. However, vegetation acts as a canopy, so that the emissivity for densely vegetated wet soil can appear similar to dry soil. New ice also has a low dielectic constant (similar to that of dry soil) and therefore has a high emissivity.

The emissivity characteristics change dramatically for heterogeneous materials that contain particles of wavelength dimensions. This is the case of multilayer (also summer melt) ice, which has voids (scattering centers) in the ice sheets due to brine depletion. Dry snow consists of ice particles that also scatter microwave radiation. The 


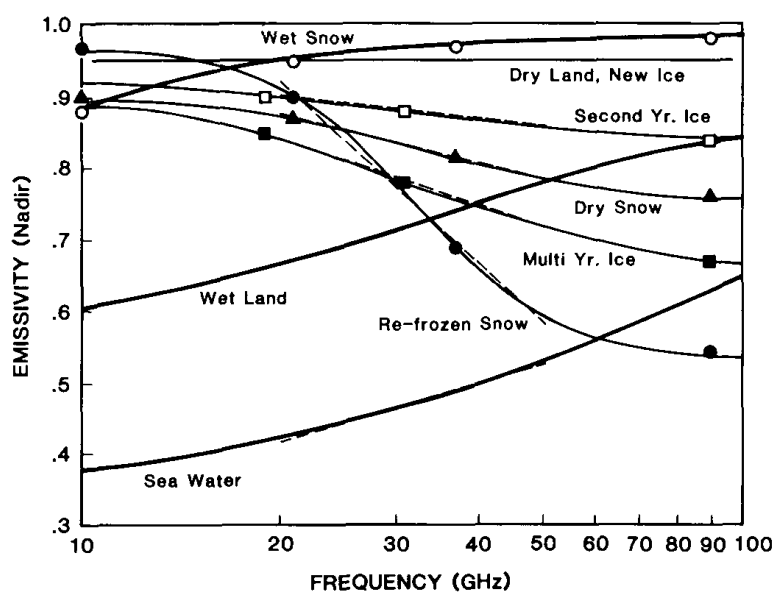

Fig. 1. Emissivity at nadir as a function of frequency for different surfaces. Solid curves are based on theoretical models and experimental measurements $(\mathrm{O}=$ wet snow, $\boldsymbol{\Delta}=$ dry snow, $\boldsymbol{\bullet}=$ refrozen snow $\square=$ second year ice, $\mathbf{\square}=$ multiyear ice). Dashed lines represent linear fits over the $20-50 \mathrm{GHz}$ range.

TABLE I

Coefficients for Four Parameter Emissivity Model (5)

\begin{tabular}{|c|c|c|c|c|c|}
\hline & SURFACE & $\varepsilon_{0}$ & $\varepsilon_{\infty}$ & wo $(\mathrm{GHz})$ & $k$ \\
\hline \multirow{3}{*}{ ICE } & New & .95 & .95 & -- & -1 \\
\hline & Second Yr. & .93 & .83 & 31 & 2 \\
\hline & Multiyear & .92 & .64 & 31 & 2 \\
\hline \multirow{3}{*}{ SNOW } & Wet & .76 & .99 & 9 & 2 \\
\hline & Dry & .90 & .75 & 33 & 3 \\
\hline & Refrozen & .97 & .53 & 32 & 4 \\
\hline
\end{tabular}

scattering effects result in a decrease in emissivity with increasing frequency by scattering some of the upwelling radiation out of the field of view. Note from Fig. 1 that the emissivity of sea ice also decreases with increasing concentration and size of brine pockets (i.e., ice age). Similarly, for dry snow the emissivity decreases with increasing snow density and crystal size (i.e., refrozen snow). The strong scattering in the case of refrozen snow results in emissivities less than that of water for high frequencies. However, when snow melts, its structure and electromagnetic properties change markedly. As temperatures increase to the melting point the effective dielectric constant of the melting ice increase to that of water coated ice particles, which mainly absorb microwave radiation. Moist snow therefore becomes highly emissive, having an emissivity varying between that of wet and dry land depending on the melting conditions [12], [13].

\section{A Problem of Uniqueness}

From Fig. 1, as well as (5), it is reasonable to expect that the frequency variation of emissivity generally contains no more than four degrees of freedom. Furthermore, many applications involve frequencies between approxi-
TABLE II

Coffricients for Two Parameter Emissivity Model, (6)

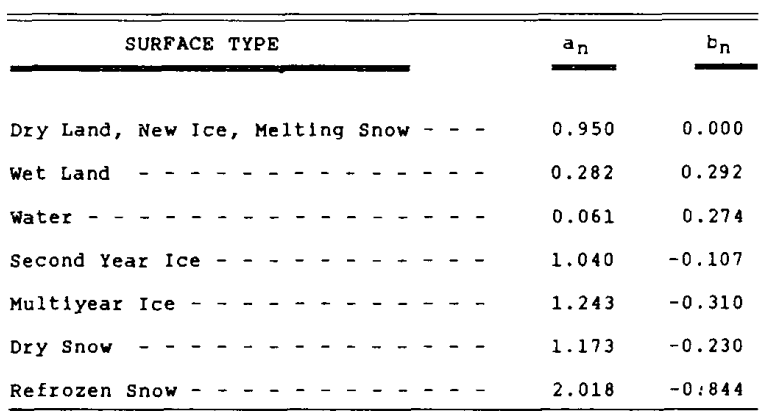

mately 20 and $50 \mathrm{GHz}$ where the emissivity can be described by only two parameters, viz.

$$
\epsilon_{n}(w)=a_{n}+b_{n} F(w) .
$$

A logarithmic function of frequency (i.e., $F(w)=$ $\log (w))$ appears adequate for representing the data shown in Fig. 1. This form of (6) is shown by the dashed lines in Fig. 1, which accurately fit the emissivity curves for the above frequency range. The parameters $a_{n}$ and $b_{n}$ are listed in Table II for the different surfaces.

Equation (6) indicates that three or more frequency measurements are linearly dependent and the emissivity can be interpolated between any two measurements. Consequently, the inversion of (4a) for $f_{n}$ becomes singular for a matrix of higher order than two (i.e., $W_{n}(w 1)$, $W_{n}(w 2)$, and $W_{n}(w 3)$, are linearly dependent). Therefore, a maximum of three surfaces can be separated $\left(f_{0}\right.$, $\left.f_{1}, f_{2}\right)$ using two linearly independent measurements $\left(E_{1}\right.$, $\left.E_{2}\right)$. The solution for $f_{n}$ is obtained using (4) and limiting the series to two terms.

$$
\begin{aligned}
& f_{1}=\frac{W_{2}(w 2) E(w 1)-W_{2}(w 1) E(w 2)}{W_{1}(w 1) W_{2}(w 2)-W_{1}(w 2) W_{2}(w 1)} \\
& f_{2}=-\frac{W_{1}(w 2) E(w 1)-W_{1}(w 1) E(w 2)}{W_{1}(w 1) W_{2}(w 2)-W_{1}(w 2) W_{2}(w 1)} \\
& f_{0}=1-f_{1}-f_{2} .
\end{aligned}
$$

This solution is applicable when the field of view contains three distinct surfaces of unknown concentration. The dual channel measurements can then separate the features within an individual pixel element. A notable example using (7) involves the determination of sea ice concentration. In polar regions the SMMR measurements at 18 and $37 \mathrm{GHz}$ are used to derive the fractional amount of open water, new ice, and multilayer sea ice within a footprint [14], [15].

The above formulation is also applicable when the field of view contains a single unknown surface. In this case the measured emissivity is given by (3a), where the functions $\epsilon_{n}(w)$ are the a priori values for three different surfaces (e.g., open water, wet land, dry land). The amplitudes $f_{n}$ are determined from the emissivity measurements according to (7). If either of the a priori functions are 
representative of the actual surface then the amplitudes derived from the measurements would be close to zero or unity depending on the surface being observed. This procedure of identifying surfaces is based on the proximity of the dual-frequency measurements to the a priori estimates. A graphical presentation of the technique is given below.

The use of dual-frequency measurements to identify different surfaces is graphically illustrated in Fig. 2(a). Shown is the emissivity at nadir for water, wet soil, snow, and ice at 24 and $31 \mathrm{GHz}$. These channels are part of the Advanced Microwave Sounding Unit (AMSU) to be launched in the 1990's [16]. The emissivity at $31 \mathrm{GHz}$ is plotted against the emissivity difference at 31 and $24 \mathrm{GHz}$, where the values were computed using the emissivity models described in Section III. Variations in the emissivity for water and wet soil are due to the temperature dependence of the dielectric constant for liquid water [9], [10]. As discussed below, the emissivities and brightness temperatures are computed using eight different temperature and water vapor profiles. Four of the eight cases have surface temperatures below freezing $(263,266,269$, $270 \mathrm{~K}$ ) and are used in conjunction with the snow and ice surfaces. The five highest surface temperatures $(270,278$, $280,282,286 \mathrm{~K}$ ) are used when computing the emissivity and brightness temperatures for the water and wet soil surfaces. Also, the integrated water vapor varies from 0.3 $\mathrm{cm}$ for the coldest temperature to $1.8 \mathrm{~cm}$ for the warmest atmosphere.

In Fig. 2(a) the different surfaces are readily identified by their unique position in the scatter diagram. Note that the largest separation between the surfaces occurs along the horizontal axis. This axis represents the emissivity slope with frequency and varies considerably between the absorbing (water, wet land) and scattering surfaces (snow, ice). The variation along the vertical axis provides additional separation between the surfaces. A mixed pixel effect would fill the region between surfaces with additional points. As mentioned in Section II, a more complete evaluation must include the effects of atmospheric attenuation on the "derived" surface emissivity as well as the uncertainties in the a priori emissivities or model inaccuracies. The atmospheric effects are simulated using the radiative transfer equation to compute the brightness temperatures at the two frequencies.

Brightness temperature calculations require information on atmospheric temperature and water vapor as well as emissivity [1]. As mentioned above, eight different temperature and water vapor profiles are used in computing the clear atmosphere brightness temperatures. Cloudy simulations are obtained by adding a layer of liquid water absorption and performing the radiative transfer calculations using the same eight temperature and water vapor soundings. The cloud layer contains liquid water varying between $0.15 \mathrm{~mm}$ for the coldest temperature profile to $0.40 \mathrm{~mm}$ for the warmer temperatures.

To represent the satellite results, the emissivities at 24 and $31 \mathrm{GHz}$ were obtained by dividing the simulated mea-

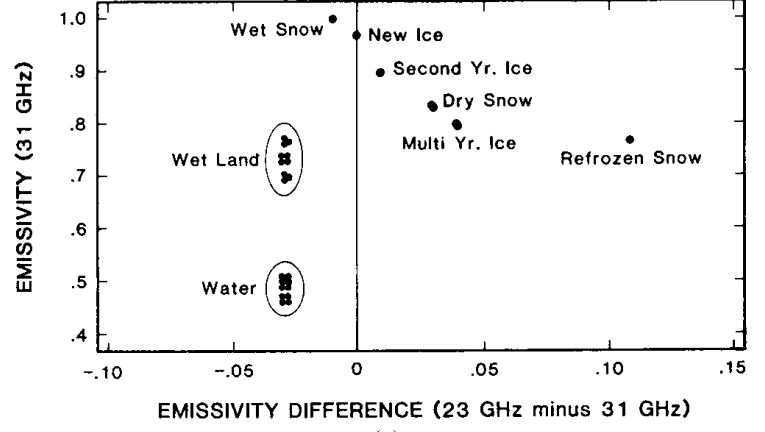

(a)

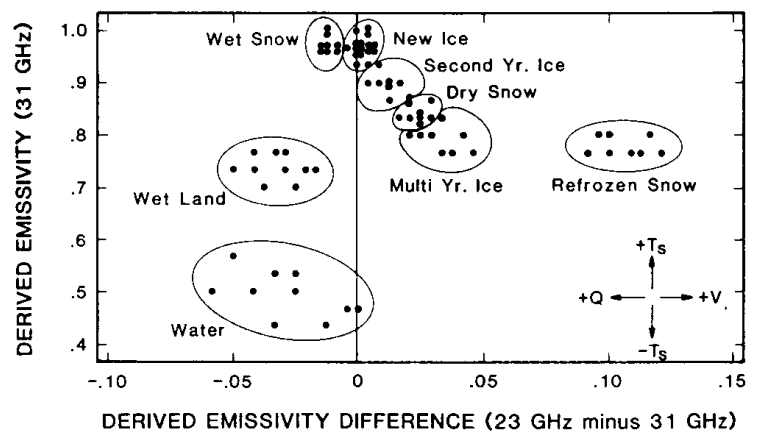

(b)

Fig. 2. (a) Emissivity at $31 \mathrm{GHz}$ versus the difference in emissivity between 24 and $31 \mathrm{GHz}$ for the surfaces shown in Fig. 1. (b) Simulations of the derived emissivity at $31 \mathrm{GHz}$ versus the difference in the derived emissivity at 24 and $31 \mathrm{GHz}$. The single points and small clusters in Fig. 2(a) become expanded in different directions due to the effects of cloud liquid water $(Q)$, water vapor $(V)$, and surface temperature $\left(T_{s}\right)$ variations on the derived emissivity.

surements by the surface temperature. Since the emissivity at each frequency is obtained using a single channel, no corrections are made for cloud and water vapor effects. However, rather than use a mean value of surface temperature, a more accurate estimate is obtained using the lower sounding AMSU channel at $53.6 \mathrm{GHz}$ [16]. This channel is unaffected by the surface emissivity variations, but responds to the atmospheric temperature near $700 \mathrm{mb}$, which correlates with the surface temperature to about 4 $\mathrm{K}$ (rms). The results are plotted in Fig. 2(b) using the same coordinate system shown in Fig. 2(a). However, unlike the previous figure, the emissivities are now derived quantities and contain the uncertainty due to atmospheric variables. Errors resulting from the effects of water vapor, clouds, and surface temperature on the derived emissivity are evident by comparing the two figures.

In Fig. 2(a) the single points corresponding to snow and ice and the small clusters due to wet soil and water are dispersed in Fig. 2(b) due to the effects of water vapor $(V)$, cloud liquid water $(Q)$, and surface temperature $\left(T_{s}\right)$ on the derived emissivity. This reduces the separation among the surfaces although there is still adequate discrimination for most of the surfaces. As indicated in Fig. 2(b), the three perturbing quantities $\left(Q, V, T_{s}\right)$ distort the 
clusters in different directions. The elongated pattern for each cluster is due to the high correlation of errors among adjacent channels for atmospheric effects. Also note the reduced atmospheric effects for the higher emissivity surfaces. These points were mentioned earlier and shall be examined further in the next section. Also studied are the effects of uncertainties in the a priori emissivity on the problems of surface identification and channel selection.

\section{Errors Due to Inaccuracy in Measurement}

In (7) the accuracy of $f_{n}$ depends on the measurement error contained in $E(w)$ as well as the uncertainty of the a priori emissivity within $W_{n}(w)$. To determine these error effects on the fractional amount the quantities given by $(4 b)$ and $(4 c)$ are rewritten

$$
\begin{gathered}
E(w)=\left[\epsilon(w)-\epsilon_{0}(w)\right]+\left[\delta \epsilon(w)-\delta \epsilon_{0}(w)\right] \\
W_{n}(w)=\left[\epsilon_{n}(w)-\epsilon_{0}(w)\right]+\left[\delta \epsilon_{n}(w)-\delta \epsilon_{0}(w)\right]
\end{gathered}
$$

where $\epsilon(w)$ and $\epsilon_{n}(w)$ are the error-free values and $\delta \epsilon(w)$ and $\delta \epsilon_{n}(w)$ are the errors associated with the emissivity measurement and a priori values, respectively. As discussed in Sections II and IV, the error in the emissivity measurement can depend on the variations in transmittance by clouds and water vapor. This was shown to produce a strong correlation in the frequency dependence of $\delta \in(w)$. Errors in the a priori emissivity are also considered to be frequency dependent although the errors are more uncertain and will be considered more random than the emissivity measurements.

To simplify the problem only one of the three surfaces $\left(\epsilon_{2}\right)$ is considered to have an uncertainty in emissivity (i.e., $\delta \epsilon_{0}=\delta \epsilon_{1}=0$ ). This is a reasonable assumption since generally one of the two surfaces consists of water, whose emissivity is well known, and the other is either dry land, melting snow, or new ice, whose emissivity is near unity. The third surface having emissivity $\epsilon_{2}$ is the most variable, and may be multilayer ice, wet land, or refrozen snow. For example, in the case of sea ice the emissivity of multiyear ice has a much larger uncertainty than that of water and new ice.

Based on (7) and (8), the error in the fractional amount $\delta f_{n}$ becomes

$$
\begin{aligned}
& \delta f_{2}=-\frac{W_{1}(w 2) \delta E(w 1)-W_{1}(w 1) \delta E(w 2)}{W_{1}(w 1) W_{2}(w 2)-W_{1}(w 2) W_{2}(w 1)} \\
& \delta f_{1}=\frac{W_{2}(w 2) \delta E(w 1)-W_{2}(w 1) \delta E(w 2)}{W_{1}(w 1) W_{2}(w 2)-W_{1}(w 2) W_{2}(w 1)} \\
& \delta f_{0}=-\left(\delta f_{1}+\delta f_{2}\right)
\end{aligned}
$$

where the terms $W_{n}(w)$ are given by $8(\mathrm{~b})$ with $\delta \epsilon_{0}=\delta \epsilon_{1}$ $=0$. The uncertainties due to the a priori emissivity $\delta \epsilon_{2}(w)$ and measurement error $\delta \epsilon(w)$, are contained in the terms $\delta E(w 1)$ and $\delta E(w 2)$, which are

$$
\begin{aligned}
& \delta E(w 1)=\delta \epsilon(w 1)-\delta \epsilon_{2}(w 1) f_{2} \\
& \delta E(w 2)=\delta \epsilon(w 2)-\delta \epsilon_{2}(w 2) f_{2} .
\end{aligned}
$$

These quantities contain the major error contribution in (9), although the error in the a priori emissivity is also included in the terms $W_{2}(w)$.

To obtain a more explicit form, showing the frequency dependence of the fractional error, the surface emissivity is approximated by (6). The error in the a priori emissivity is due to uncertainties in the slope $\delta b$ and intercept $\delta a$ parameters, so that we define

$$
\begin{aligned}
& a_{2}=\bar{a}_{2}+\delta a \\
& b_{2}=\bar{b}_{2}+\delta b
\end{aligned}
$$

where

$$
\delta \epsilon_{2}(w)=\delta a+\delta b F(w)
$$

and $\bar{a}_{2}, \bar{b}_{2}$ are the error-free emissivity parameters. For an arbitrary surface, there is no reason to expect a strong correlation between $\delta a$ and $\delta b$. Therefore, the error in the a priori emissivity represents a source of random error and will have a large influence on the characteristics of the fractional error.

Substituting ( $8 b$ ) into (9) and using (11c), the fractional error can be written as

$$
\delta f_{n}=S_{n} \frac{\delta E(w 1)-\left[A_{n}+F(w 1)\right] Q}{A_{2}-A_{1}}
$$

where

$$
Q=\frac{\delta E(w 2)-\delta E(w 1)}{F(w 2)-F(w 1)}
$$

and the coefficients $A_{n}$ and $S_{n}$ are given by

$$
\begin{array}{lll}
A_{2}=\frac{a_{1}-a_{0}}{b_{1}-b_{0}} & A_{1}=\frac{a_{2}-a_{0}}{b_{2}-b_{0}} \quad A_{0}=\frac{a_{2}-a_{1}}{b_{2}-b_{1}} \\
S_{2}=\frac{-1}{b_{2}-b_{0}} & S_{1}=\frac{1}{b_{1}-b_{0}} \quad S_{0}=-S_{1}-S_{2} .
\end{array}
$$

The frequency dependence of the fractional error is contained in the $Q$ factor, which is a function of the frequency separation as well as the difference in the emissivity errors. However, as shown below, the fractional error given by (12a) contains a number of unusual features that are very sensitive to the error characteristics of $\delta E(w)$.

Although it would appear that the $Q$ values and the fractional errror would increase rapidly for small frequency separation, the two quantities generally remain small even as the frequency separation approaches zero. This is clearly the case when the frequency dependence of the error terms $\delta E(w)$ varies more rapidly than the emissivity function $F(w)$. As demonstrated below, under these conditions the fractional error is minimal for small frequency separation and "increases for large separation." An un- 
expected result also occurs when the errors $\delta E(w)$ are identical for the two channels, or when they vary in the same manner as the emissivity, i.e., $F(w)$. In both instances the fractional error is "independent of the frequency separation." Another unusual result occurs when $Q=\delta E(w 1) /\left(A_{n}+F(w 1)\right)$. This condition results in zero fractional error, but requires a perfect balance between the various quantities, which is highly unlikely even for one of the three surfaces.

These situations involving specific values of $Q$ represent rare events. Any random, uncorrelated error component contained in $Q$ would reduce their effect and significantly change the fractional error characteristics. As mentioned previously, a major source of uncorrelated errors arises from uncertainties in the a priori emissivity parameters (see (10), (11)). This random contribution is introduced in $\delta E(w)$

$$
\delta E(w)=\delta \bar{E}(w)+\delta \tilde{E}(w)
$$

where $\bar{\delta} E(w)$ is the correlated errors (e.g., atmospheric effects) and $\delta E(w)$ is the random noise component. In general the errors increase with frequency and are modeled as

$$
\begin{aligned}
\delta \bar{E}(w) & =\delta \bar{E}(w 1)(w / w 1)^{p} \\
\left\langle\delta \tilde{E}(w)^{2}\right\rangle & =\left\langle\delta \tilde{E}(w 1)^{2}\right\rangle(w / w 1)^{2 q}
\end{aligned}
$$

where the parameters $p$ and $q$ generally have values near unity, but will be varied in the examples to follow.

From (12) and (13), the root mean squared fractional error can be written as the combination of two terms

$$
\sqrt{\left\langle\delta f_{n}^{2}\right\rangle}=\sqrt{\delta \bar{f}_{n}^{2}+\left\langle\delta \bar{f}_{n}^{2}\right\rangle}
$$

where $\delta \bar{f}_{n}^{2}$ is given by (12) where only the correlated error component (14a) is used. This results in the equation

$$
\begin{aligned}
& \frac{\delta \bar{f}_{n}^{2}}{\delta \bar{E}(w 1)^{2}} \\
& \quad=S_{n}^{2} \frac{\left(\left[A_{n}+F(w 2)\right]-\left[A_{n}+F(w 1)\right](w 2 / w 1)^{p}\right)^{2}}{\left(A_{2}-A_{1}\right)^{2}(F(w 2)-F(w 1))^{2}} .
\end{aligned}
$$

The second term $\left\langle\tilde{\delta} f_{n}^{2}\right\rangle$ is obtained from (12) but the error is considered uncorrelated so that

$$
\begin{aligned}
& \frac{\left\langle\delta \tilde{f}_{n}^{2}\right\rangle}{\left\langle\delta \tilde{E}(w 1)^{2}\right\rangle} \\
& =S_{n}^{2} \frac{\left[A_{n}+F(w 2)\right]^{2}+\left[A_{n}+F(w 1)\right]^{2}(w 2 / w 1)^{2 q}}{\left(A_{2}-A_{1}\right)^{2}(F(w 2)-F(w 1))^{2}} .
\end{aligned}
$$

To illustrate the behavior of (15), the fractional error, as well as the individual components $\delta \bar{f}_{n}$ and $\sqrt{\left\langle\delta \tilde{f}_{n}^{2}\right\rangle}$, are plotted as a function of frequency for the case when the three surfaces have the characteristics of new ice $(n=$ $0)$, water $(n=1)$, and multiyear ice $(n=2)$. Emissivity parameters $\left(a_{n}, b_{n}\right)$ are obtained from Table II with $F(w)$ $=\log (w)$. The lowest-frequency, $w 1$, is set to $19 \mathrm{GHz}$ and the second frequency is increased up to $50 \mathrm{GHz}$. The results shown in Fig. 3 consider the errors in $\delta \bar{E}(w)$ and $\delta \tilde{E}(w)$ to be equal, having a value of 0.01 at all frequencies (i.e., $p=q=0$ ). In accordance with (15), the results for larger errors are proportionally greater.

Note that the fractional error is dominated by the random error component (17), whose magnitude becomes very large for small frequency separation. The correlated error component (16) is smaller than the random error term and constant with frequency for the case when $p=0$. Also observe that the smallest fractional error occurs for the water surface, which is the same magnitude as that obtained for the total ice concentration.

As a second example, the error quantities are made frequency dependent by setting $p=q=1$. Results of this more realistic simulation are shown in Fig. 4. As in the previous example, the fractional error is primarily defined by the random error component. However, now the correlated error term becomes significant with higher frequency separation. Note also that the random error component decreases, while the correlated error term increases for larger frequencies. This results in a minimum fractional error, where its lowest value occurs over a broad range of frequencies. It appears that a second frequency of about $35 \mathrm{GHz}$ provides adequate separation to identify the three surface types. These results will vary somewhat depending on the errors in $\delta \bar{E}$ and $\delta \tilde{E}$ and on their frequency dependence (i.e., $p, q$ ). However, the major differences arise when considering other classes of surfaces.

Calculations were performed for the case where the three surfaces consist of dry land, water, and wet soil. Fig. 5 shows the results for frequency independent errors $(p=q=0)$, while Fig. 6 contains the results when $p=$ $q=1$. Note that the emissivity model of dry land is the same as new ice so that only the emissivity of the third surface (wet soil) is different from the previous case. This makes an interesting comparison since the spectral responses of these surfaces all have slopes of the same sign. Compared to the sea ice case, this reduces the signatures among the three surfaces, resulting in larger fractional errors. As before, a second channel frequency exceeding 35 $\mathrm{GHz}$ does not appear to add much improvement.

In general, the smallest fractional errors occur when only correlated errors exist in the emissivity measurement and $a$ priori emissivity $(\delta \tilde{E}=0)$. This results in the greatest separation among surface types, and is further improved when the errors are the same for both channels $(p=0)$. However, neither of these conditions are met in actual practice. Uncertainties, particularly in the a priori emissivity, result in a random error component that provides the major error. As indicated below, techniques for addressing this problem require the use of additional surface information. 


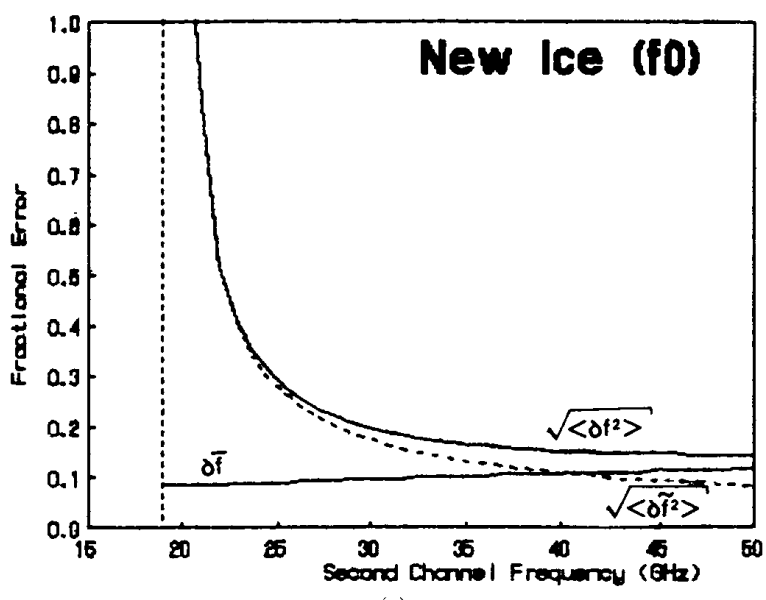

(a)

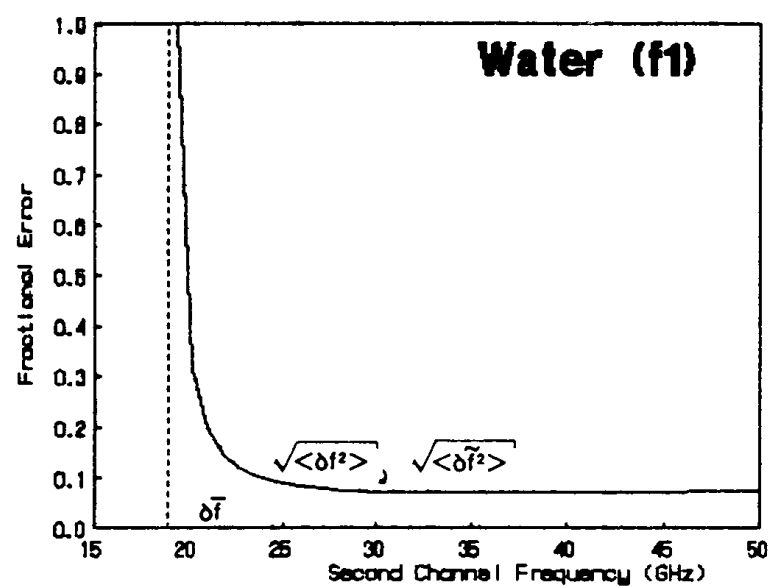

(b)

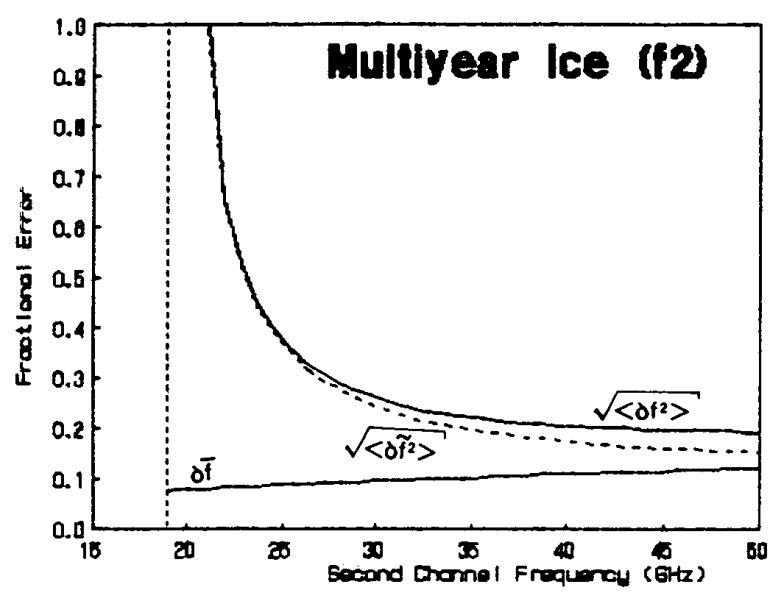

(c)

Fig. 3. Errors in the fractional amount (rms) for new ice, water, and multiyear ice, $\sqrt{\left\langle\delta f^{2}\right\rangle}$. Also shown are the individual components due to correlated, $\delta f$, and uncorrelated errors, $\sqrt{\left\langle\phi f^{2}\right\rangle}$, in the emissivity measurements and a priori data. The correlated and uncorrelated emissivity errors are set to 0.01 and are considered frequency independent, i.e., $p$ $=q=0($ see $(14),(15))$

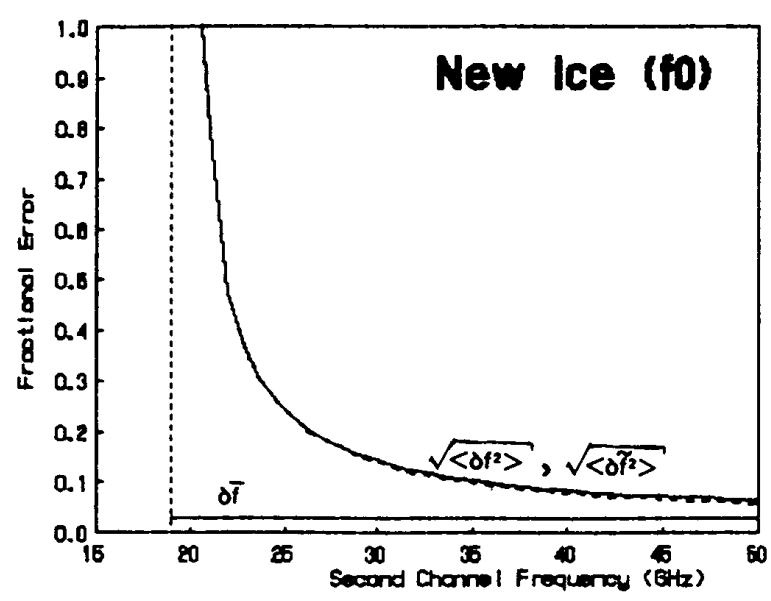

(a)
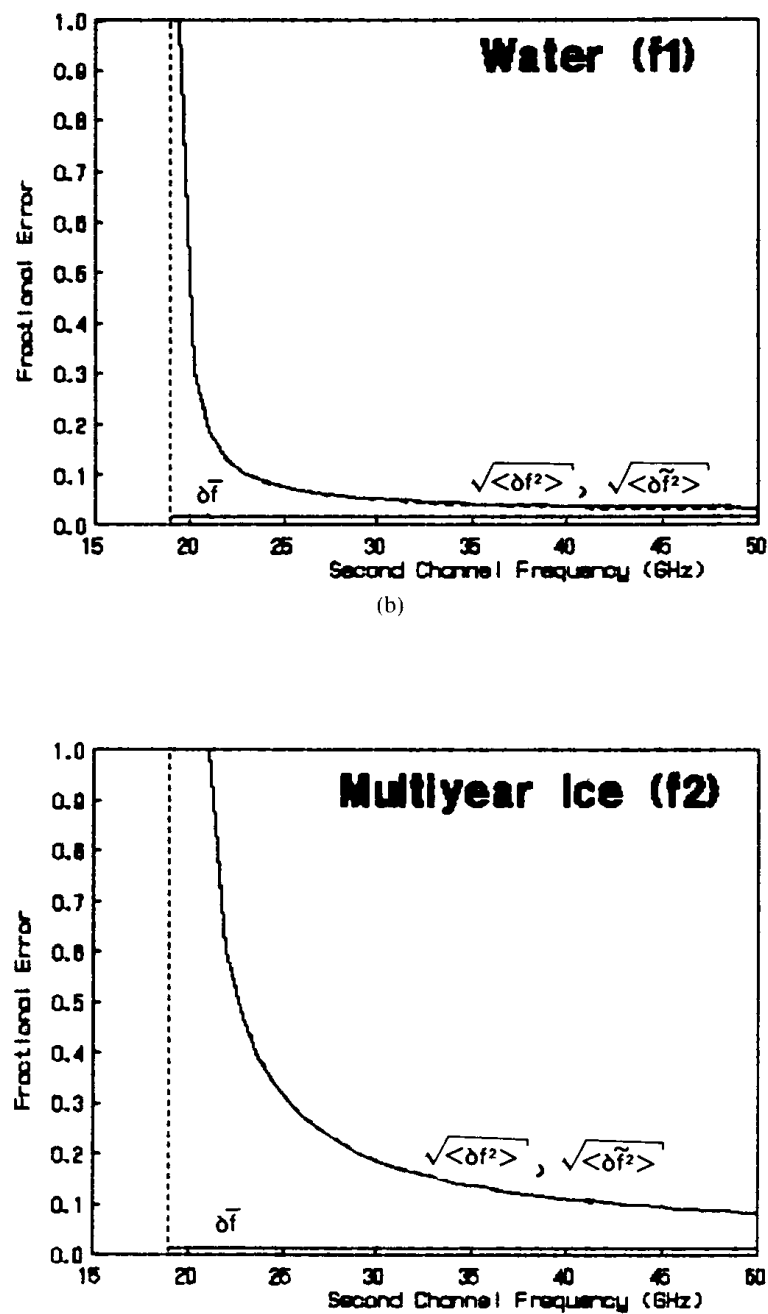

(c)

Fig. 4. Same as in Fig. 3 except that the emissivity errors are considered frequency dependent such that $p=q=1$. 


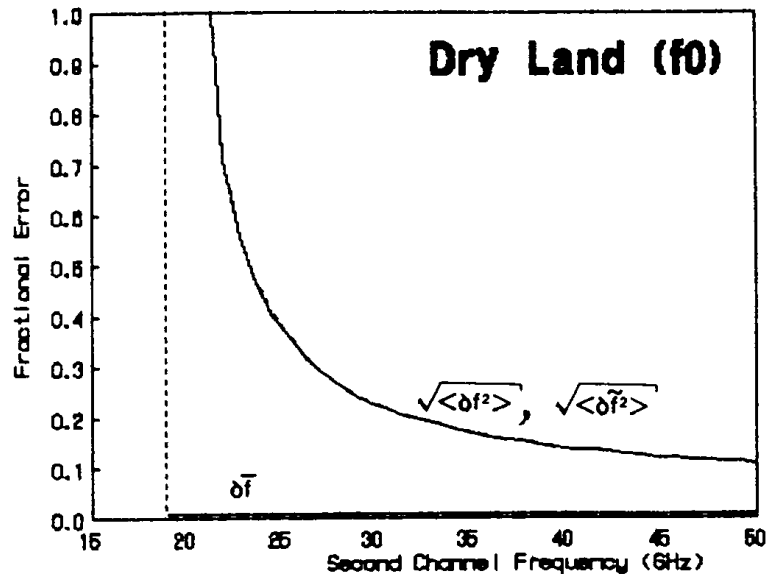

(a)

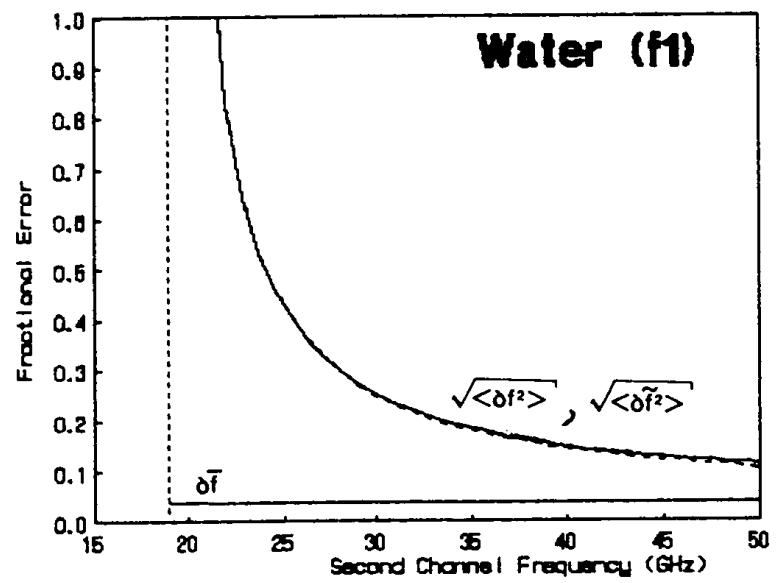

(b)

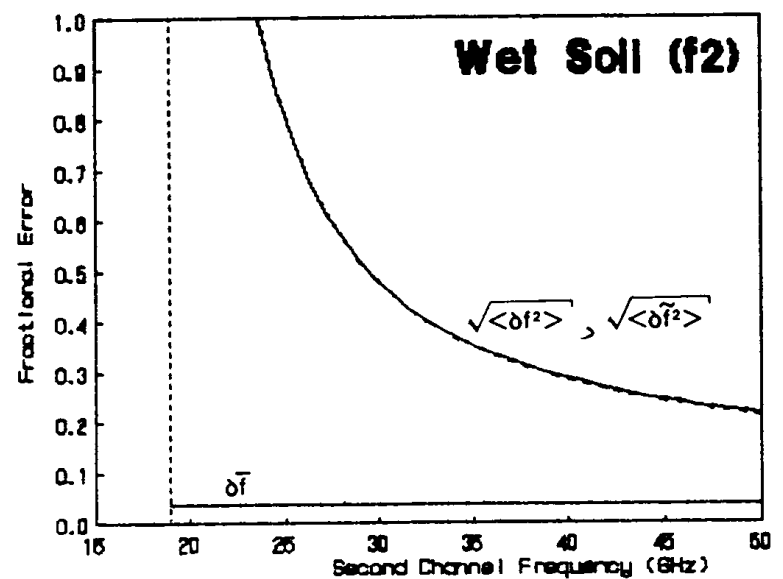

(c)

Fig. 5. Errors in the fractional amount (rms) for dry land, water, and wet soil, $\sqrt{\left\langle\phi f^{2}\right\rangle}$. Also shown are the individual components resulting from correlated, $\delta f$, and uncorrelated errors, $\sqrt{\left\langle\phi f^{2}\right\rangle}$, in the emissivity measurements and a priori data. The correlated and uncorrelated emissivity errors are set to 0.01 and are considered frequency independent, i.e., $p$ $=q=0(\operatorname{see}(14),(15))$.

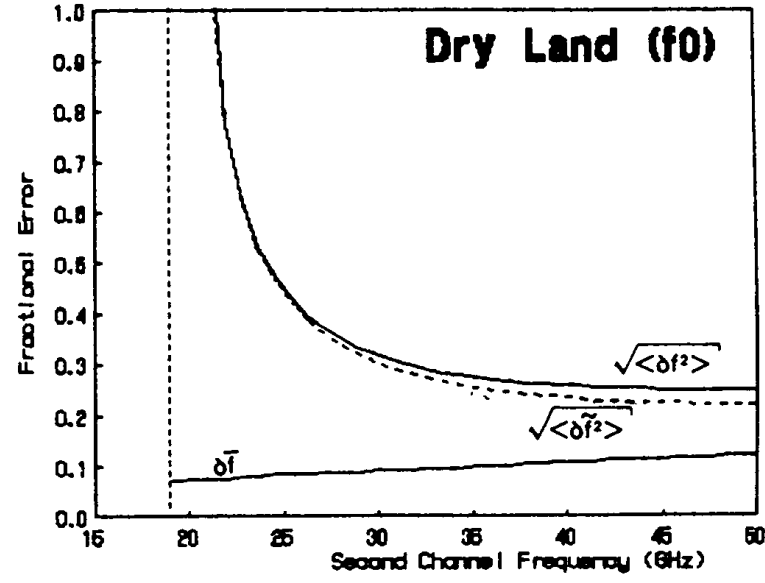

(a)

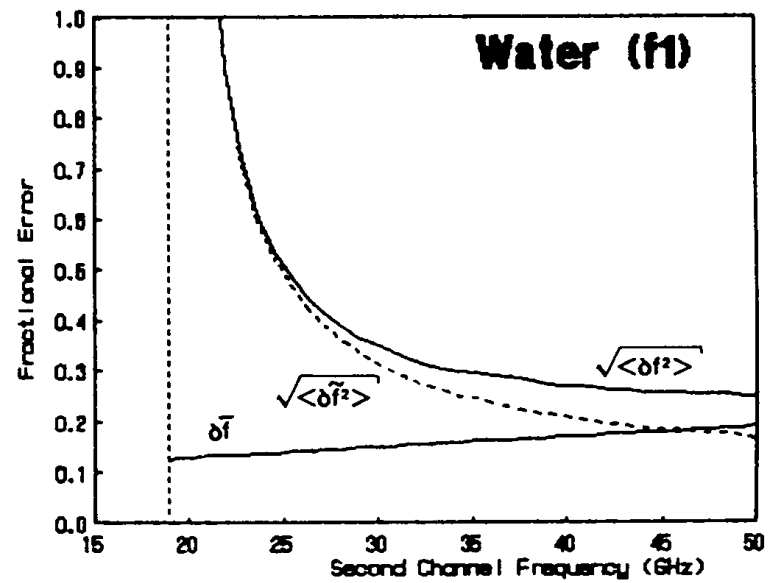

(b)

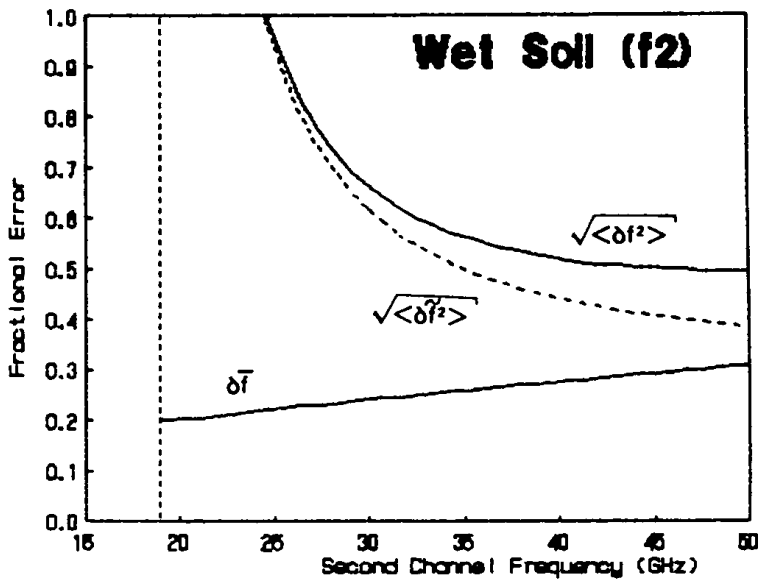

Fig. 6. Same as in Fig. 5 except that the emissivity errors are considered frequency dependent such that $p=q=1$. 


\section{Summary AND CONClusions}

Passive microwave measurements show a strong potential for identifying surfaces and retrieving surface parameters. Unlike the response at optical wavelengths the microwave response is strongly dependent on the water content and liquid or ice phase of surfaces. The different spectral characteristics of water, land, snow, and sea ice are used for surface identification.

Various techniques are available to identify surface features from multichannel satellite measurements. For example, a library search technique could be used to match the microwave measurements at different frequencies to specific surface types. Although the library technique is easily expanded to include different channels and additional data sources, it is difficult to examine the various interactions among channels and data types. To visualize the problem of surface identification a graphical procedure was described in Section IV using dual frequency measurements. However, to obtain quantitative results, an analytical solution was obtained by expressing the surface emissivity as a linear combination of known emissivities.

The analytical formulation was developed by considering the "mixed pixel" problem where many surfaces are contained within the viewing area. At frequency $w$ the emissivity measurement $\epsilon(w)$ depends on the fractional amounts $f_{n}$ and a priori emissivities $\epsilon_{n}(w)$ where $\epsilon(w)=$ $\Sigma \epsilon_{n}(w) f_{n}$. In applications involving surface identification the fractional amounts act as discriminants to identify the most likely surface among the a priori candidates. In principle the fractional amounts can be obtained using multispectral single polarization measurements of emissivity. However, due to the limited spectral characteristics of the emissivity for natural surfaces the maximum number of distinguishable surfaces is reduced to three. The fractional amounts are obtained using dual frequency emissivity measurements, where the effects due to errors in the measurements and a priori data were analyzed.

The accuracy of the fractional amounts is limited by the accuracy of the emissivity measurements and a priori values. In general the errors in the emissivity measurements are strongly correlated for the two channels, resulting in relatively small errors in the fractional amounts. However, the a priori emissivity values represent a source of random error and produce large errors in the fractional amounts. Examples in the case of sea ice and wet land surfaces illustrate the characteristics of the fractional error due to these different error sources. Based on these examples, the use of dual frequency measurements at about 19 and $35 \mathrm{GHz}$ is shown to offer the best compromise for measuring surface features.

In addition to the use of spectral information, temporal information can be very useful for improving the surface estimate. Unlike instantaneous point measurements, continuous observations can provide data on surface conditions (e.g., melting of snow and ice) and filter out the effects of precipitation on the measurements. It is also im- portant to utilize the infrared surface temperature measurements and visible observations of surface reflectivity. Techniques must be developed to incorporate these data together with the microwave measurements. The use of microwave polarization measurements can also serve as additional predictors in the retrieval method although their uniqueness compared to spectral measurements has not been evaluated. Not to be overlooked is the use of additional measurements around $90 \mathrm{GHz}$, which could not be examined at this time. However, the recently launched Special Sensor Microwave Imager (SSMI) instrument on the Defense Meteorological Satellite Program (DMSP) satellite contains an $85-\mathrm{GHz}$ channel. Its use for surface observations must await further analysis by researchers.

\section{ACKNOWLEDGMENT}

The author wishes to acknowledge G. Reinhardt, R. Ambroziak, and D. Crosby of NESDIS for their help and useful suggestions during the preparation of this paper.

\section{REFERENCES}

[1] F. T. Ulaby, R. K. Moore, and A. K. Fung, Microwave Remote Sens ing. Active and Passive, vol. 1. Reading, MA: Addison Wesley, 1981.

[2] - Microwave Remote Sensing, Active and Passive, vol. 3. Reading, MA: Addison Wesley, 1986.

[3] N. C. Grody, D. G. Gray, C. S. Novak, J. S. Prasad, M. Piepgrass, and C. A. Dean, "Temperature soundings from the DMSP microwave sounder," in Advances in Remote Sensing Retrieval Methods Hampton, VA: Deepak, 1985, pp. 249-265.

[4] N. C. Grody, A. Gruber, and W. C. Shen, "Atmospheric water content derived from the Nimbus-6 scanning microwave spectrometer over the tropical pacific,"'J. Appl. Meteorol., vol. 19, pp. 986-996, 1980

[5] R. R. Ferraro, N. C. Grody, and J. A. Kogut, "Classification of geophysical parameters using passive microwave satellite measurements," IEEE Trans. Geosci. Remote Sensing, vol. GE-24, no. 6 , pp. 1008-1013, Nov. 1986.

[6] R. W. Spencer, W. S. Olson, W. Rongzhang, D. Martin, J. A. Weinman, and D. A. Santek, "Heavy thunderstorms observed over land by the Nimbus- 7 scanning multichannel microwave radiometer," $J$. Clim. Appl. Meteorol., vol. 22, pp. 1041-1046, 1983.

[7] D. T. Eppler, L. D. Farmer, and A. W. Lohanik, "Classification of sea ice types with single band $(33.6 \mathrm{GHz})$ aerborne passive microwave imagery," J. Geoph. Res., vol. 91, pp. $10661-10695,1986$.

[8] L. Tsang, J. A. Kong, and R. T. Shin, Theory Of Microwave Remote Sensing. New York: Wiley, 1985

[9] J. R. Wang and T. T. Schmugge, "An empirical model for the complex dielectric permittivity of soils as a function of water content,' IEEE Trans. Geosci. Remote Sensing, vol. GE-18, pp. 288-295, 1980.

[10] L. A. Klein and C. T. Swift, "An improved model for the dielectric constant of sea water at microwave frequencies," IEEE Trans. Antennas Propagat., vol. AP-25, pp. 104-114, 1977.

[11] J. P. Hollinger, B. E. Troy, R. O Ramseier, K. W Asmus, M. F. Hartman, and C. A. Luther, "Microwave emission from high artic sea ice during freeze-up,' J. Geophys. Res., vol. 89, pp. 8104-8122, 1984

[12] C. Matzler, E. Shanda, and W. Good, "Toward the definition of optimum sensor specifications for microwave remote sensing of snow," IEEE Trans. Geosci. Remote Sensing, vol. GE-20, pp. 57-66, 1982.

[13] K. F. Kunzi, S. Patil, and H. Rott, "Snow-cover parameters retrieved from Nimbus-7 scanning multichannel microwave radiometer (SMMR) data," Rev. Geophys. Space Phys., vol. 22, pp. 452-467, 1984

[14] E. Svendsen, K. Kloster, B. Farrelly, O. M. Johannessen, J. A. Johannessen, W. J. Cambell, P. Gloerson, D. Cavalieri, and C. Matzler, "Norwegian remote sensing experiment: Evaluation of the Nimbus 7 scanning multichannel microwave radiometer for sea ice research,"'J. Geophys. Res., vol. 88, pp. 2781-2791, 1983. 
[15] C. T. Swift and D. J. Cavalieri, "Passive microwave remote sensing for sea ice research," EOS, Trans. American Geophys. Union., vol. 66 , no. 49,1985

[16] N. C. Grody, "Determination of surface and atmospheric parameters using the advanced microwave sounding unit," in Proc. 3rd Conf. Satellite Meteorology Oceanography (Anaheim, CA), pp. 100-104, Jan. 31-Feb. 5, 1988

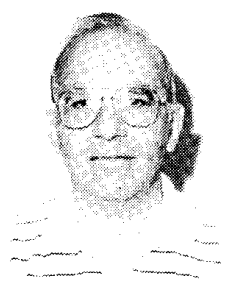

Norman C. Grody was born in the Bronx, NY, on March 6, 1940. He received the B.E.E. degree from the City College of New York in 1962, and the M.E.E. and Ph.D. degrees in electrical engineering from New York University in 1964 and 1972 , respectively.

Between 1966 and 1967 he was with C.B.S. Laboratories, Stamford, CT, where he performed analysis of electo-optic crystals for use as ligh modulators and beam deflectors. From 1967 to 1968 he worked at ITT Defense and Communi- cations Division, Nutley, $\mathrm{NJ}$, on the design and fabrication of an $X$-band matstrip antenna for a portable communication system. During 1968 to 1971 he was a Research Associate at New York University in the Electrical Engineering Department and performed research on the linear and nonlinear response of a magnetoplasma column to impulse excitation. Since 1972 he has been with the National Environmental Satellite, Data and Information Service, of NOAA where he has served as coinvestigator for the microwave experiments aboard the Nimbus- 5 and Nimbus-6 NASA satellites as well as the more recent instruments aboard the NOAA and DMSP satellites. His primary interest concerns the application of microwave radiometry for providing temperature and water vapor soundings, atmospheric winds, precipitation, and land parameters.

Dr. Grody is a member of Eta Kappa Nu. 